\title{
Application of hyperspherical close-coupling method to antiproton collisions with muonic hydroge
}

\author{
Akinori Igarashi ${ }^{1}$ and Nobuyuki Toshima ${ }^{2}$ \\ ${ }^{1}$ Faculty of Engineering, University of Miyazaki, Miyazaki 889-2192, Japan \\ ${ }^{2}$ Institute of Materials Science, Graduate School of Pure and Applied Sciences, \\ University of Tsukuba, Tsukuba, 305-8573, Japan
}

\begin{abstract}
The cross sections for $p^{-}+\left(H \mu^{-}\right)_{1 s}(H=p, d, t)$ are calculated in the hyperspherical close-coupling method for collision energies below the $\left(\mathrm{Hp}^{-}\right)_{n=4}$ threshold. The results show good convergence with respect to basis size. The formation cross section of $\left(\mathrm{Hp}^{-}\right)_{n=2}$ is dominant and two orders of magnitude larger than that of $\left(\mathrm{Hp}^{-}\right)_{n=1}$ below the $\left(\mathrm{Hp}^{-}\right)_{n=3}$ threshold, while the $\left(\mathrm{Hp}^{-}\right)_{n=3}$ formation cross-section exceeds that for $\left(\mathrm{Hp}^{-}\right)_{n=2}$ formation above the $\left(\mathrm{Hp}^{-}\right)_{n=3}$ threshold. Resonance parameters are also calculated below thresholds of $\left(\mathrm{Hp}^{-}\right)_{n=2-4}$.
\end{abstract}

\section{Introduction}

The hyperspherical close-coupling (HSCC) method is a powerful tool for studying bound states and scattering states for three-body systems [1]. The HSCC method has been extended to general three-body systems such as $e^{ \pm}$-atom collisions $[2,3]$, ion-atom collisions $[4,5,6]$, muon-transfer $[7,8,9]$, three-body recombination $[12,13]$, reactive scattering $[10,11]$, and nucleon systems [14], with no restrictions on the masses of the particles. Notable advantages of the HSCC method are that i) the convergence with respect to the basis set is generally fast for low energy collisions, since the basis, adiabatic channel functions, is obtained by diagonalizing the adiabatic Hamiltonian including all interactions among particles; ii) the adiabatic potential curves give useful information about resonances and couplings among the channels; and iii) non-local potentials are completely absent in the scattering equations even for systems in which rearrangement channels must be considered.

We have applied the HSCC method to three-body systems consisting of a nucleus $Z(=1,2,3)$ and muonic hydrogen in order to calculate cross sections for muon transfer $[15,16]$ and spin-flip process $[17,18]$. Recent progress in experimental techniques to produce slow antiproton $\left(p^{-}\right)$beams opens up new research possibilities in a range of fields, including atomic physics and nuclear physics [19]. In applying HSCC to muonic hydrogen targets, we consider $p^{-}$collisions with muonic hydrogen in the ground state, namely, $p^{-}+\left(H \mu^{-}\right)_{1 s}$, where $\mu^{-}$denotes a negative muon and $H$ stands for hydrogen isotopes $(p, d, t)$. At a low-energy limit of the collision, the hydrogenic atom $\left(H p^{-}\right)$ is formed in $n=1$ and 2. The notation $n$ denotes the principal quantum number of the hydrogenic atoms. The cross sections are calculated for collision energies below the $\left(\mathrm{Hp}^{-}\right)_{n=4}$ threshold to see the contribution of $\left(\mathrm{Hp}^{-}\right)_{n=3}$ formation. Resonances below the $\left(H p^{-}\right)_{n=2-4}$ thresholds are also calculated.

Unfortunately, no work on the $p^{-}+\left(H \mu^{-}\right)_{1 s}$ collisions has not been reported so 
far. While, mass-scaled $p^{-}+\mathrm{H}(1 s)$ collisions, similar to the $p^{-}+\left(p \mu^{-}\right)_{1 s}$ collision, were treated by Esry and Sadeghpour [20] $\left(m_{p} / m_{e}=7.4796\right.$ and 17.824) and by Hesse, Le, and Lin [21] $\left(m_{p} / m_{e}=100\right)$, where $m_{p}$ is the scaled mass of $p^{ \pm}$and $m_{e}$ is the electron mass. The $p^{-}+\left(p \mu^{-}\right)_{1 s}$ collision corresponds to a mass scaled $p^{-}+\mathrm{H}(1 s)$ collision with $m_{p} / m_{e}=8.88$.

Muon atomic units (m.a.u), where the muon mass $\left(m_{\mu}=206.769 m_{e}\right)$ is set to unity, and $\hbar=e=1$ are used throughout this paper unless otherwise stated.

\section{HSCC calculation}

The internal motion of three particles is described by the hyperradius $\rho$ and five angular variables $\Omega$ in hyperspherical coordinates. In the HSCC method [15], the scattering wave function is expanded by the product of the radial function $F_{i}(\rho)$ and the adiabatic channel functions $\varphi_{i}(\rho, \Omega)$ as

$$
\Psi^{J \Pi}(\rho, \Omega)=\sum_{i}^{N} \frac{F_{i}(\rho)}{\rho^{5 / 2}} \varphi_{i}(\rho, \Omega)
$$

for each partial-wave $J$ and parity $\Pi$. Inserting this expansion into the Schrödinger equation, we obtain coupled differential equations for $\left\{F_{i}\right\}$ :

$$
\begin{aligned}
& \left(-\frac{1}{2 \mathcal{M}} \frac{d^{2}}{d \rho^{2}}+U_{i}(\rho)-E\right) F_{i}(\rho) \\
& +\sum_{j} W_{i j}(\rho) F_{j}(\rho)=0
\end{aligned}
$$

where $U_{i}(\rho)$ is the adiabatic potential associated with $\varphi_{i}, E$ is the total energy, and $W_{i j}(\rho)$ represents non-adiabatic coupling. The mass parameter $\mathcal{M}$ is taken to be the reduced mass of $\left(\mathrm{Hp}^{-}\right)$. $W_{i j}$ is sharply peaked around the avoided crossing of the adiabatic potentials. A slight modification is made to solve the coupled equations for computational convenience, namely a hybrid expansion of the adiabatic and diabatic channel functions is adopted [15]. Since the system is an arrangement of a charged particle and a hydrogenic atom in the asymptotic region, the dipole representation [22] is appropriate for channels in the Jacobi coordinates. The wave function in Eq. (1) is matched with the scattering boundary conditions in the Jacobi coordinates at sufficiently large hyperradius, $\rho_{M}$, to extract the scattering matrix. The cross sections are stable to within a few $\%$ for $\rho_{M}>400$.

\section{Results}

Hesse et al. [21] calculated S-wave cross sections for the mass scaled $p^{-}+\mathrm{H}(1 s)$ collision with $m_{p}=m_{p^{-}}=100 m_{e}$ using their HSCC method including 46 channels and 10 channels selected by a diabatization technique. The mass scaled $p^{-}+\mathrm{H}(1 s)$ collision is analogous to $p^{-}+\left(H \mu^{-}\right)_{1 s}$ collisions. Before showing the results, we compare the cross sections of Hesse et al. and those of the present HSCC calculation where 35 channels 
dissociating into $p^{-}+\mathrm{H}(1 s)$ and $e^{-}+\left(p p^{-}\right)_{n=1-8}$ are coupled. As shown in Fig. 1, the present HSCC calculation successfully reproduces the cross sections of Hesse et al. Hence, the present calculation is expected to be valid for $p^{-}+\left(H \mu^{-}\right)_{1 s}$ collisions. In Fig. 1, the 10-channel calculation of Hesse et al. slightly differs from the other calculations for small cross sections of $\left(p p^{-}\right)_{n=5}$.

For $p^{-}+\left(H \mu^{-}\right)_{1 s}$ collisions, HSCC calculations are carried out for partial-waves $J=0-4$ for collision energies below the $\left(\mathrm{Hp}^{-}\right)_{n=4}$ formation threshold. Two different basis sets are adopted for each collision: basis set A includes channels which describe fragmentations into $p^{-}+\left(H \mu^{-}\right)_{1 s}$ and $\mu^{-}+\left(H p^{-}\right)_{n=1-3}$ at large $\rho$, and basis set $\mathrm{B}$ includes channels which describe fragmentation into $\mu^{-}+\left(H p^{-}\right)_{n=4}$ in addition to the channels of basis set A.

\subsection{Cross sections}

S-wave potential curves for $\left(p^{-}, H=(p, d, t), \mu^{-}\right)$systems are shown in Fig. 2. Each potential curve converges to an atomic energy of $\left(H p^{-}\right)_{n}$ or $\left(H \mu^{-}\right)_{n}$ as $\rho \rightarrow \infty$ and the corresponding channel describes the fragmentation $\mu^{-}+\left(H p^{-}\right)_{n}$ or $p^{-}+\left(H \mu^{-}\right)_{n}$. The potential curves are similar among the three isotope systems. The orders of the asymptotic energies are $\left(\mathrm{Hp}^{-}\right)_{n=1},\left(\mathrm{Hp}^{-}\right)_{n=2},\left(\mathrm{H}^{-}\right)_{n=1},\left(\mathrm{Hp}^{-}\right)_{n=3},\left(\mathrm{Hp}^{-}\right)_{n=4}, \cdots$ from the bottom. The potential curve for the initial channel $p^{-}+\left(H \mu^{-}\right)_{1 s}$ is located between those converging to the $\left(\mathrm{Hp}^{-}\right)_{n=2}$ and $\left(\mathrm{Hp}^{-}\right)_{n=3}$ thresholds at $\rho \rightarrow \infty$. It is conjectured from Fig. 2 that the $p^{-}+\left(H \mu^{-}\right)_{1 s}$ channel strongly couples with the $\mu^{-}+\left(H p^{-}\right)_{n=2}$ channels. The reduced masses of the $\left(\mathrm{Hp}^{-}\right)$and $\left(H \mu^{-}\right)$atoms increase with the mass number of $H$, and its binding energies increases likewise. Since the muon mass is much smaller than those of $H$ and $p^{-}$, the energy difference between $\left(H \mu^{-}\right)_{1 s}$ and $\left(H p^{-}\right)_{n=2}$ and between $\left(H \mu^{-}\right)_{1 s}$ and $\left(H p^{-}\right)_{n=3}$ increases or decreases, respectively, with an increase of the mass number of $H$, owing to the reduced mass.

Figure 3 shows the cross section $(\sigma)$ multiplied by the velocity $(v)$ below the $\left(H p^{-}\right)_{n=3}$ formation threshold against the center-of-mass collision energy $\left(E_{c}\right)$. For the low energy region, $v \times \sigma[23]$ behaves as $v$ for elastic scattering and is constant for $\left(\mathrm{Hp}^{-}\right)_{n=1-2}$ formation. Basis sets $\mathrm{A}$ and $\mathrm{B}$ give similar results, which shows good convergence. The formation cross section of $\left(\mathrm{Hp}^{-}\right)_{n=2}$ is approximately two orders of magnitude larger than that of $\left(\mathrm{Hp}^{-}\right)_{n=1}$. The partial cross sections in dipole states show that $\left(\mathrm{Hp}^{-}\right)_{n=2}$ formation occurs through the lowest adiabatic states of the manifold at low energies, which is consistent with HSCC calculations for mass-scaled $p^{-}+\mathrm{H}$ collisions $[20,21]$.

Figure 4 shows the cross sections for basis set B for collision energies near the $\left(H p^{-}\right)_{n=3}$ thresholds and below the $\left(\mathrm{Hp}^{-}\right)_{n=4}$ thresholds. The $\left(\mathrm{Hp}^{-}\right)_{n=3}$ formation cross section is finite at the threshold owing to the degeneracy of $\left(\mathrm{Hp}^{-}\right)$atoms $[22,23]$. The overall energy-dependencies are similar for the three collision systems. Resonance structures appear below the thresholds. Above the $\left(\mathrm{Hp}^{-}\right)_{n=3}$ thresholds, the formation cross sections for $\left(\mathrm{Hp}^{-}\right)_{n=3}$ increase above those for $\left(\mathrm{Hp}^{-}\right)_{n=2}$. 
In Fig. 5, we show the partial-wave cross sections for the $p^{-}+\left(t \mu^{-}\right)_{1 s}$ collision in basis set $\mathrm{B}$ as an example. S-wave contributions are dominant for the low energy region and contributions from higher partial-waves increase with collision energy. For collision energies between the $\left(t p^{-}\right)_{n=3}$ and $\left(t p^{-}\right)_{n=4}$ thresholds, P- and D-wave contributions are large for $\left(t p^{-}\right)_{n=2}$ formation, while D- and F-wave contributions are important for $\left(t p^{-}\right)_{n=3}$ formation. Similar trends are also seen for $\left(p \mu^{-}\right)$and $\left(d \mu^{-}\right)$targets.

Figure 6 shows $\ell$ distributions, namely, $\sigma_{n \ell} / \sum_{\ell} \sigma_{n \ell}$, in $\left(H p^{-}\right)_{n=2}$ and $\left(H p^{-}\right)_{n=3}$ formation, where $\ell$ denotes the angular momentum quantum number of the $\left(H p^{-}\right)$ atom and $\sigma_{n \ell}$ is the cross section for $\left(\mathrm{Hp}^{-}\right)_{n \ell}$ formation. For the $\left(\mathrm{Hp}^{-}\right)_{n=2}$ formation, $2 \mathrm{~s}$ formation is dominant at low energies in the three collision systems, but formation of the $2 \mathrm{p}$ state predominates at higher energies. For $\left(\mathrm{Hp}^{-}\right)_{n=3}$ formation, formation of the $3 \mathrm{~d}$ state is dominant.

\subsection{Resonances}

In the asymptotic region, the present systems take the arrangements $p^{-}+\left(H \mu^{-}\right)_{n}$ and $\mu^{-}+\left(H p^{-}\right)_{n}$, namely, a charged particle and a neutral hydrogenic atom at a large distance $(R)$. The polarization potential, which decays as $1 / R^{4}$, works between a charged particle and a hydrogenic atom in the ground state. When hydrogenic atoms are in excited states, they interact through the dipole potential, which decays as $\alpha /\left(2 M R^{2}\right)$, where $M$ is their reduced mass and $\alpha$ is the dipole moment. The corresponding channels are described by the dipole states [22] which take account of the Stark effect of the hydrogenic atom in excited states. An infinite series of resonances appears below the threshold when $\alpha<-1 / 4$. We show the values of $\alpha(<-1 / 4)$ for the dipole states corresponding to the $\mu^{-}+\left(\mathrm{Hp}^{-}\right)_{n=2-4}$ thresholds in Table 1 .

The resonance parameters are derived by fitting the eigenphase sum to the BreitWigner formula [24] with a linear background. In Table 2, we show the lowermost resonances associated with the $\mu^{-}+\left(H^{-}\right)_{n=2-4}$ thresholds for partial-waves $J$. As the reduced mass of $\left(H p^{-}\right)$increases, $|\alpha|$ in Table 1 decreases for a given $J$ and the asymptotic potential curve becomes less attractive. Hence, the resonance energies below the $\mu^{-}+\left(\mathrm{Hp}^{-}\right)_{n=2-4}$ channels become shallower with an increase of the mass number of $H(=p, d, t)$.

For the energy region near the $\left(H \mu^{-}\right)_{1 s}$ threshold, a broad resonance is found below the threshold in the $\left(p^{-}, t, \mu^{-}\right)$system, but not in the $\left(p^{-}, p, \mu^{-}\right)$and $\left(p^{-}, d, \mu^{-}\right)$systems. As a model, regarding that the mass of $\mu^{-}$is much smaller than the masses of $p^{-}$and $H$, we consider the bound state problem below the $p^{-}+\left(H \mu^{-}\right)_{1 s}$ threshold by the BornOppenheimer approach where the adiabatic parameter is the distance between $p^{-}$and $H$. We solve the radial equation for each system including $H=p, d$ or $t$, where the adiabatic potential is the same but the reduced mass between $p^{-}$and $H$ is different. The existence of bound states is more probable for a system of heavier reduced mass, which explains the present result. 


\section{Summary}

We have calculated the cross sections for $p^{-}$collisions with muonic hydrogen atoms $\left(H \mu^{-}\right)_{1 s}(H=p, d, t)$ in the HSCC method. The cross section in the HSCC calculation is in good convergence with respect to basis sets. $\left(\mathrm{Hp}^{-}\right)_{n=2}$ formation is dominant below the $\left(\mathrm{Hp}^{-}\right)_{n=3}$ threshold. The cross section for $\left(\mathrm{Hp}^{-}\right)_{n=2}$ formation is about two orders of magnitude larger than that for $\left(\mathrm{Hp}^{-}\right)_{n=1}$ formation. $\left(\mathrm{Hp}^{-}\right)_{n=3}$ formation becomes larger than $\left(\mathrm{Hp}^{-}\right)_{n=2}$ formation above the $\left(\mathrm{Hp}^{-}\right)_{n=3}$ formation threshold. Resonance parameters have been obtained for the lowermost resonances below the $\left(\mathrm{Hp}^{-}\right)_{n=2-4}$ thresholds.

Collision experiments for the $p^{-}+\left(H \mu^{-}\right)_{1 s}$ collision are quite difficult. However, $(p p)^{-}$formation through the $p^{-}+\mathrm{H}(1 s)$ collision and high resolution laser spectroscopy of $\left(p p^{-}\right)$are planned [25, 26]. The population of $\left(p p^{-}\right)$after the collision is a useful parameter for subsequent experiments. Recently, the state-specified formation cross section was calculated with nonperturbative quantal calculations by Tong et al. [27, 28]. They found that the formed protonium atoms tend to be distributed in higher angular momentum and higher principle quantum number states as the collision energy increases. These trends are also seen in the present system. It would be worthwhile to calculate the state-specified formation for the $p^{-}+\mathrm{H}(1 s)$ collision by different methods to verify the accuracy of the calculations. The cross sections obtained in the present work are available as a comparison for such methods. 


\section{References}

[1] C.D. Lin, Phys. Rep. 257 (1995) 1.

[2] D. Kato and S. Watanabe, Phys. Rev. A 56, 3687 (1997).

[3] A.T. Le, M. W. Bromley, and C. D. Lin, Phys. Rev. A 71, 032713 (2005).

[4] C.N. Liu, A.T. Le, T. Morishita, B. D. Esry, and C. D. Lin, Phys. Rev. A 67, 052705 (2003).

[5] A.T. Le, C. D. Lin, L. F. Errea, L. Mendez, A. Riera, and B. Pons Phys. Rev. A 69, 062703 (2004).

[6] T.G. Lee, M. Hesse, A.T. Le, and C. D. Lin, Phys. Rev. A 70, 012702 (2004).

[7] A. Dupays, B. Lepetit, J. A. Beswick, C. Rizzo, and D. Bakalov, Phys. Rev. A 69, 062501 (2004).

[8] A. Dupays Phys. Rev. Lett. 93, 043401 (2004)

[9] A.T. Le and C. D. Lin, Phys. Rev. A 71, 022507 (2005).

[10] A. Ohsaki and H. Nakamura, Phys. Rept. 187, 1 (1990).

[11] W. Hu and G.C. Schatza, J. Chem. Phys. 125, 132301 (2006).

[12] B. D. Esry, C. H. Greene, and J. P. Burke, Jr., Phys. Rev. Lett. 83, 1751 (1999).

[13] N. P. Mehta, B. D. Esry, and C. H. Greene, Phys. Rev. A 76, 022711 (2007).

[14] E. Nielsen, D.V. Fedorov, A.S. Jensen, E. Garrio, Phys. Rep. 347373 (2001).

[15] A. Igarashi, N. Toshima, and T. Shirai, Phys. Rev. A 504951 (1994).

[16] A. Igarashi and N. Toshima, Eur. Phys. J. D 40175 (2006).

[17] A. Igarashi, I. Shimamura and N. Toshima, Phys. Rev. A 581166 (1998).

[18] A. Igarashi, M. P. Faifman, I. Shimamura, Hyp. Interact. 13877 (2001).

[19] Low Energy Antiproton Physics (LEAP'05), edited by D. Grzonka et al., AIP conference proceedings. 796 (American Institute of Physics, New York, 2005).

[20] B. D. Esry and H. R. Sadeghpour, Phys. Rev. A 6712704 (2003).

[21] M. Hesse, A. T. Le, and C. D. Lin, Phys. Rev. A 6952712 (2004).

[22] M. Gailitis and R. Damburg, Proc. Phys. Soc. 82192 (1963).

[23] H. R. Sadeghpour et al., J. Phys. B 33 R93 (2000).

[24] A. U. Hazi, Phys. Rev. A 19920 (1979).

[25] N. Kuroda et al., Phys. Rev. Lett. 94023401 (2005).

[26] Y. Yamazaki, Nucl. Instrum. Methods Phys. Res., Sect. B 154174 (1999); Hyperfine Interact. 138141 (2001).

[27] X. M. Tong, K. Hino, and N. Toshima, Phys. Rev. Lett. 97, 243202 (2006).

[28] X. M. Tong, T. Shirahama, K. Hino, and N. Toshima, Phys. Rev. A 75052711 (2007). 


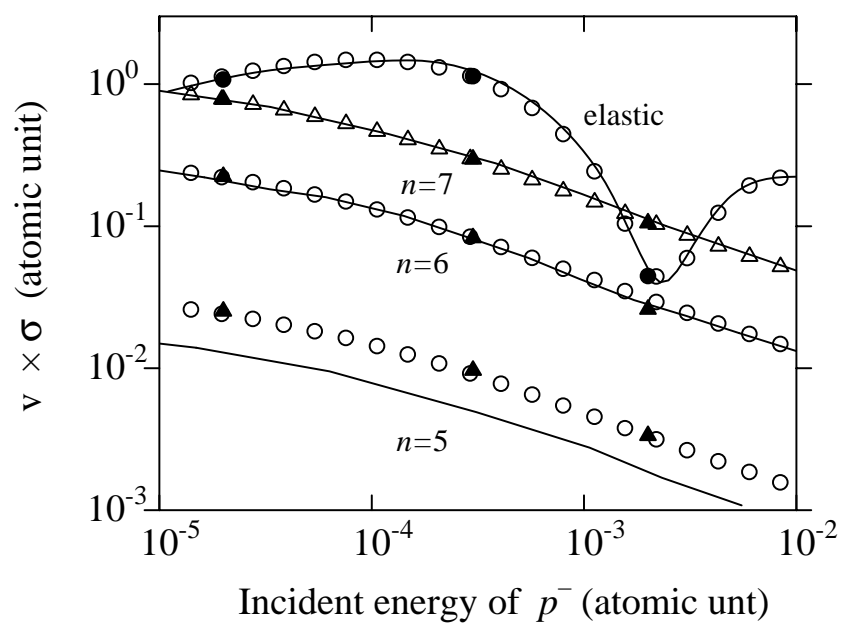

Figure 1. S-wave cross sections for elastic scattering and $\left(p p^{-}\right)_{n}$ formation in the mass-scaled $p^{-}+\mathrm{H}(1 s)$ collision with $m_{p}=m_{p^{-}}=100 m_{e}$ : 10-channel Hesse et al. [21] HSCC calculation (solid curves), 46-channel Hesse et al. HSCC calculation (full circles, full triangles), present HSCC calculation (open circles, open triangles). 

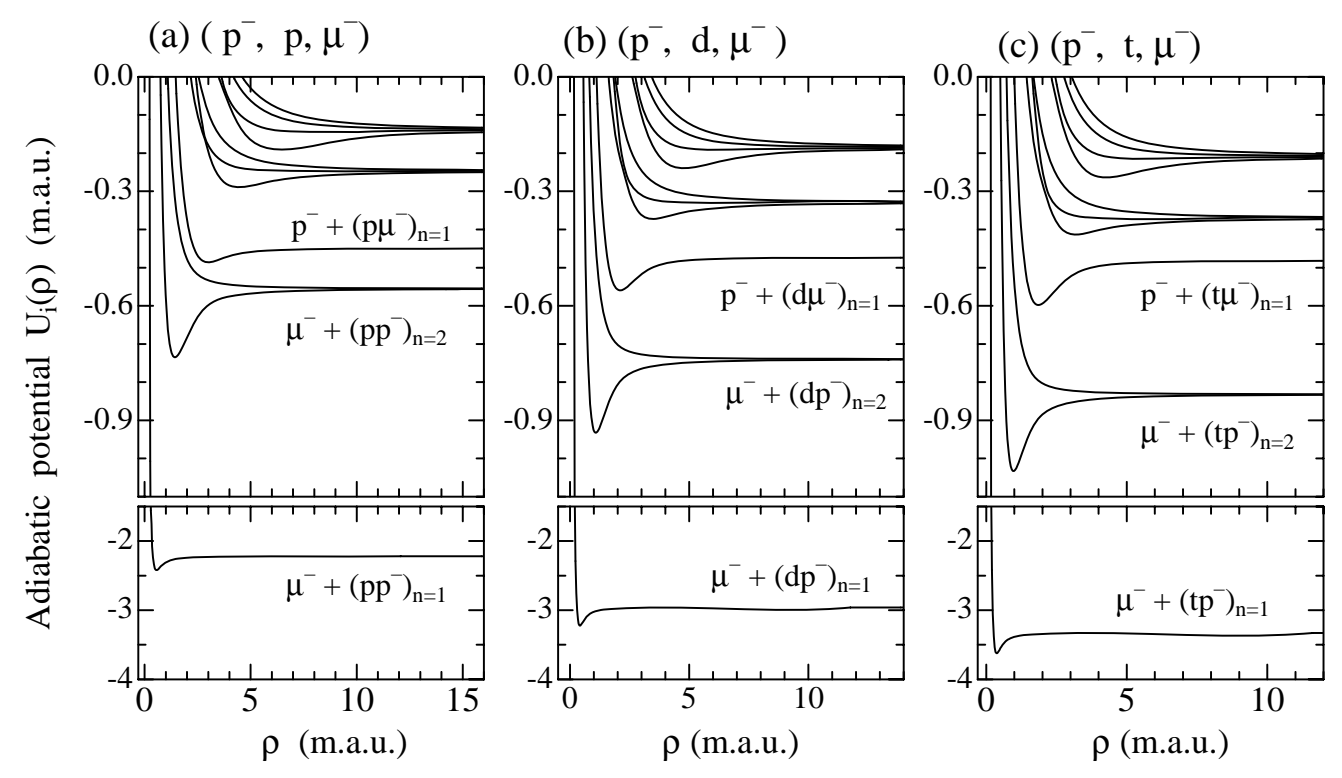

Figure 2. S-wave adiabatic potential curves for (a) $\left(p^{-}, p, \mu^{-}\right)$, (b) $\left(p^{-}, d, \mu^{-}\right)$, and (c) $\left(p^{-}, t, \mu^{-}\right)$systems. The asymptotic fragmentation described by the corresponding adiabatic channel function is indicated as $\mu^{-}+\left(p p^{-}\right)_{n}$ or $p^{-}+\left(p \mu^{-}\right)_{n}$, where $n$ denotes the principal quantum number of the hydrogenic atoms. The sets of three and four potential curves above that of $p^{-}+\left(H \mu^{-}\right)_{n=1}(H=p, d, t)$ correspond to fragmentations into $\mu^{-}+\left(H p^{-}\right)_{n=3}$ and $\mu^{-}+\left(H p^{-}\right)_{n=4}$, respectively. 


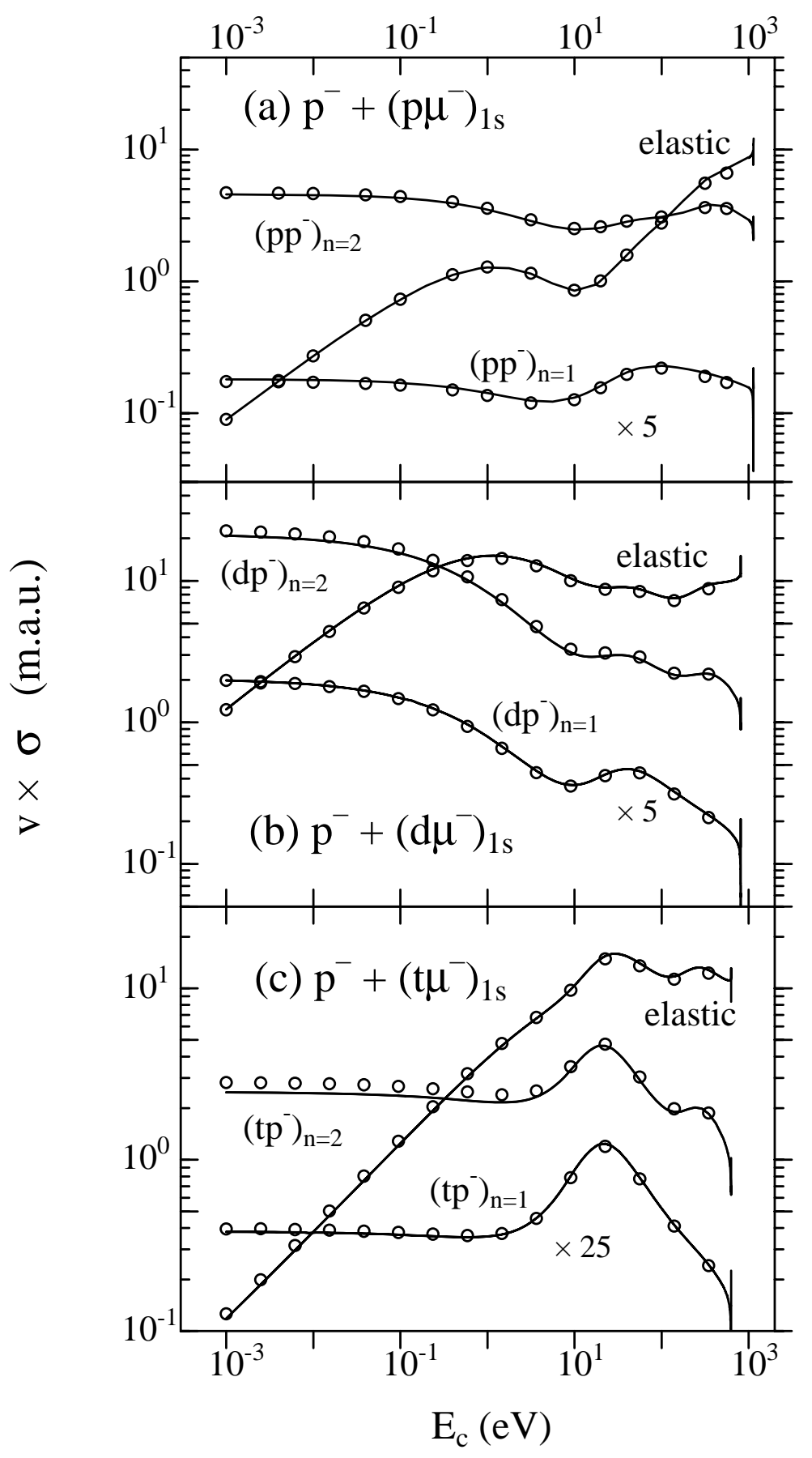

Figure 3. Cross sections $(\sigma)$ multiplied by incident velocity $(v)$ for elastic scattering and $\left(H p^{-}\right)_{n=1,2}$ formation in (a) $p^{-}+\left(p \mu^{-}\right)_{1 s}$, (b) $p^{-}+\left(d \mu^{-}\right)_{1 s}$, and (c) $p^{-}+\left(t \mu^{-}\right)_{1 s}$ collisions below the $\left(\mathrm{Hp}^{-}\right)_{n=3}$ thresholds. Basis set $\mathrm{A}$ is shown by circles and basis set $\mathrm{B}$ is shown by the solid curves. $E_{c}$ is the center-of-mass collision energy. The thresholds are $E_{c}=1140.6,813.0$, and $630.5 \mathrm{eV}$ for collisions (a), (b) and (c), respectively. 

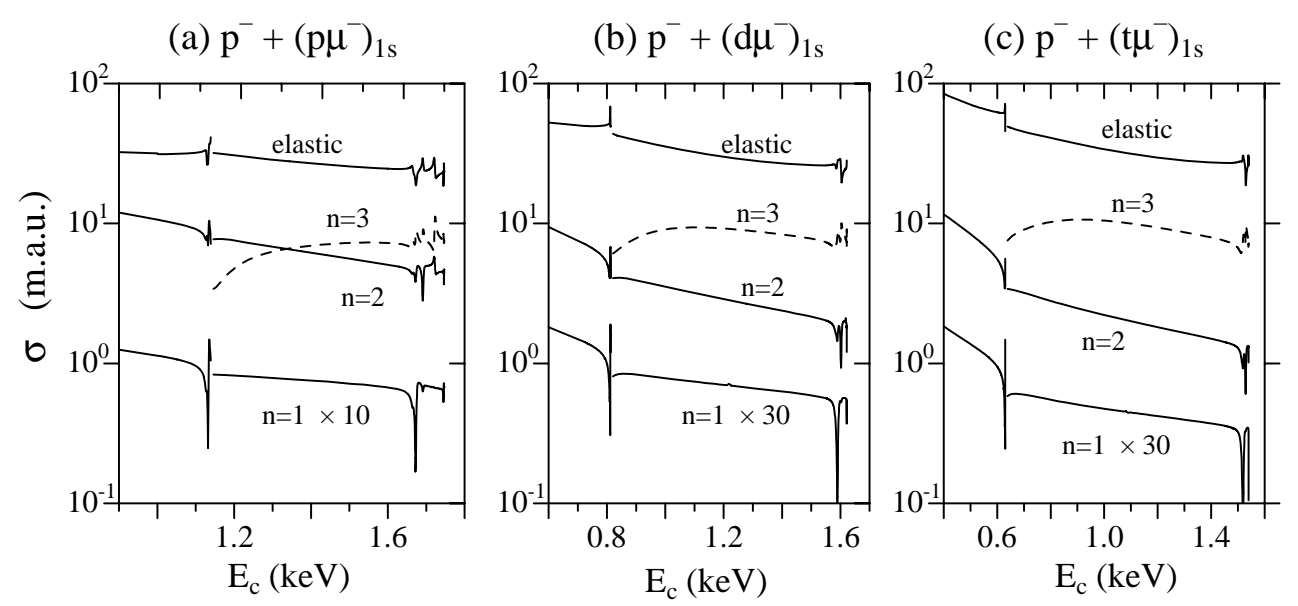

Figure 4. Cross sections for elastic scattering and $\left(\mathrm{Hp}^{-}\right)_{n}$ formations for $E_{c}$ near the $\left(H p^{-}\right)_{n=3}$ threshold and below the $\left(H p^{-}\right)_{n=4}$ threshold in (a) $p^{-}+\left(p \mu^{-}\right)_{1 s}$, (b) $p^{-}+\left(d \mu^{-}\right)_{1 s}$, and (c) $p^{-}+\left(t \mu^{-}\right)_{1 s}$ collisions. The $\left(H p^{-}\right)_{n=4}$ thresholds are 1748.3, 1622.5 and $1540.8 \mathrm{eV}$ for collisions (a), (b) and (c), respectively. 
(a)

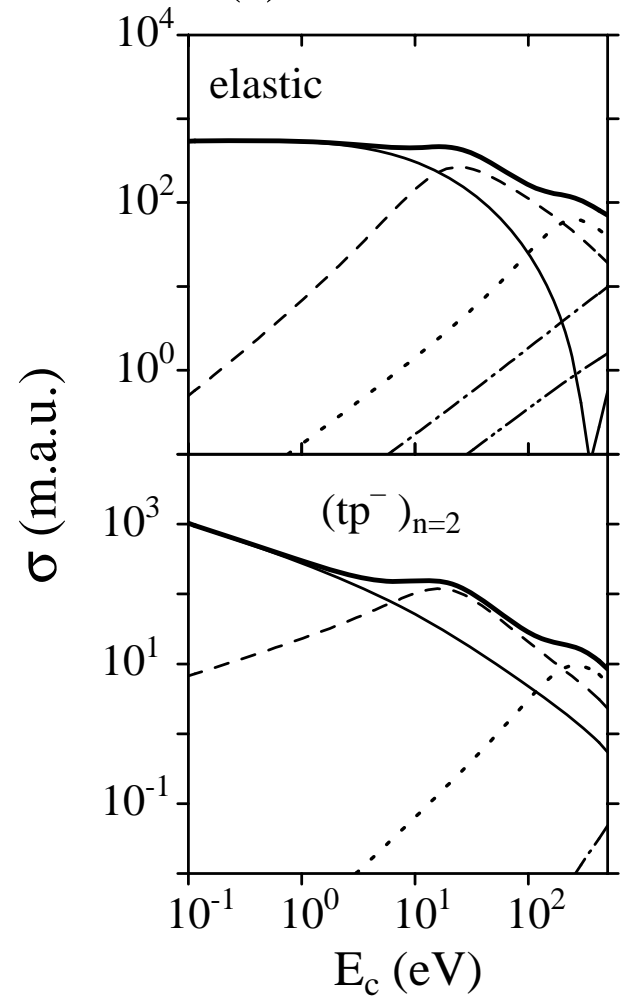

(b)

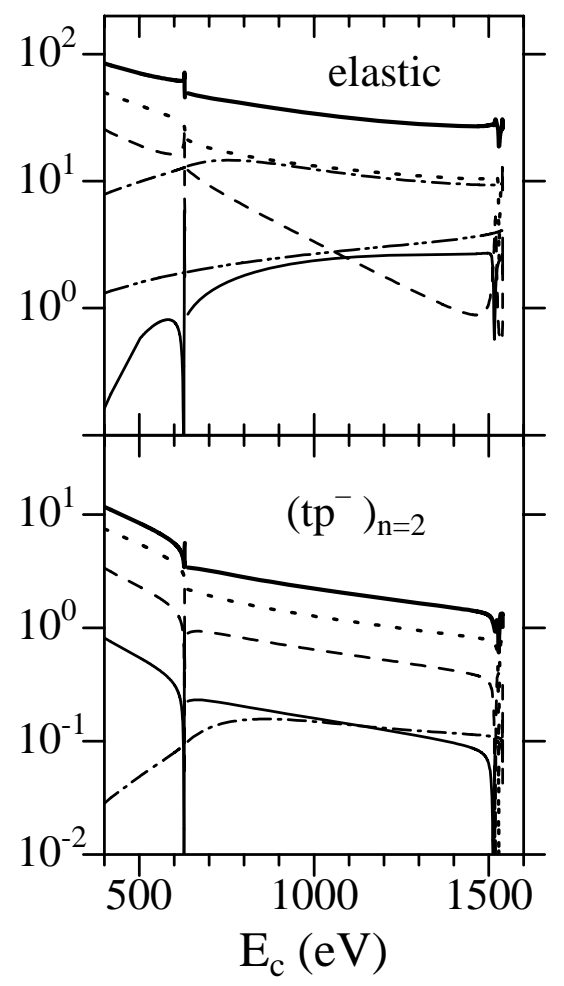

(c)

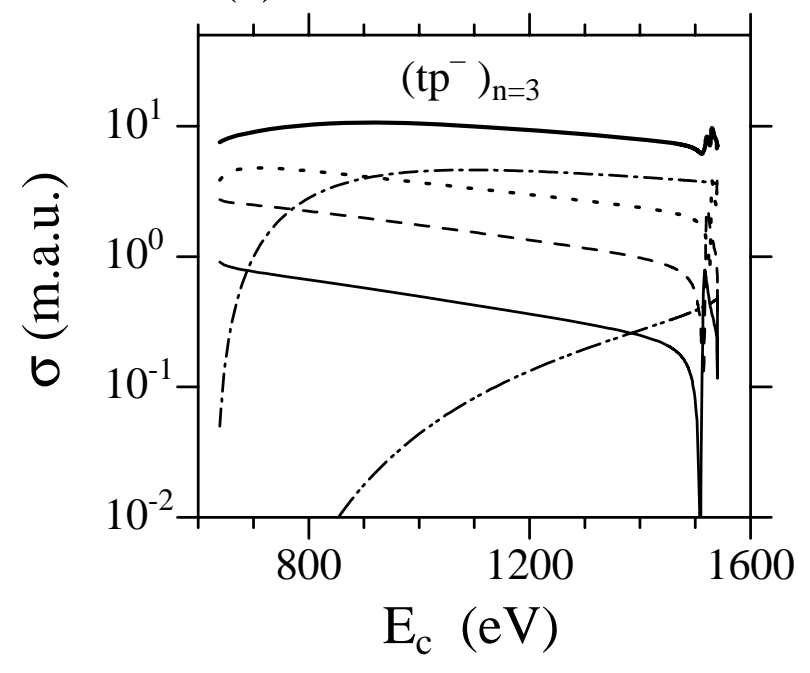

Figure 5. Partial-wave cross sections for $J=0-4$ in $p^{-}+\left(t \mu^{-}\right)_{1 s}$ collisions. Elastic scattering and $\left(t p^{-}\right)_{n=2}$ formation for (a) $E_{c}<500 \mathrm{eV}$ and for (b) $E_{c}$ near the $\left(t p^{-}\right)_{n=3}$ threshold $\left(E_{c}=630.5 \mathrm{eV}\right)$ and below the $\left(t p^{-}\right)_{n=4}$ threshold $\left(E_{c}=1540.8 \mathrm{eV}\right)$. (c) $\left(t p^{-}\right)_{n=3}$ formation below the $\left(t p^{-}\right)_{n=4}$ threshold.

$J=0$ (thin solid curve), $J=1$ (dashed curve), $J=2$ (dotted curve), $J=3$ (dot-dashed curve), $J=4$ (dot-dot-dashed curve), summed cross sections (thick solid curves). 
(a) $\mathrm{p}^{-}+\mathrm{p} \mu$
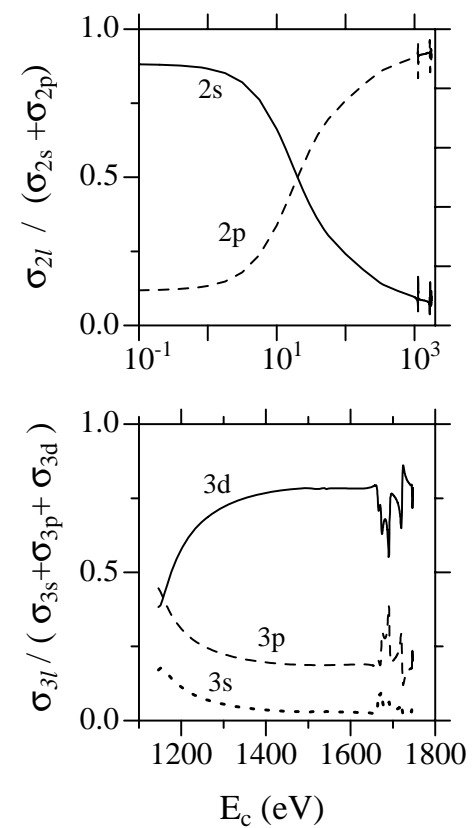

(b) $\mathrm{p}^{-}+\mathrm{d} \mu$
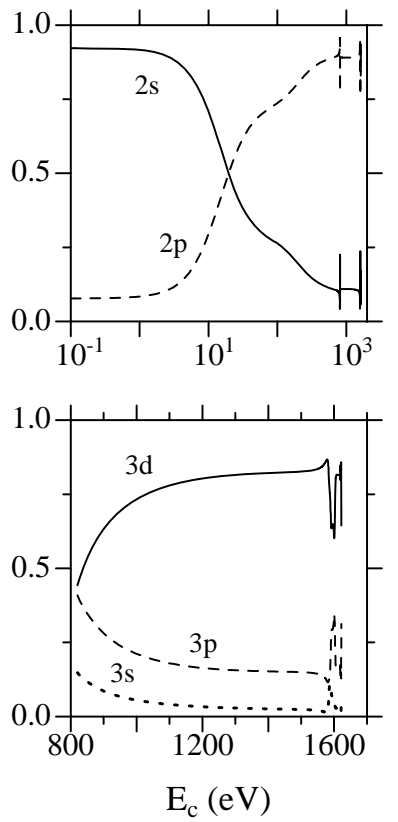

(c) $\mathrm{p}^{-}+\mathrm{t} \mu$
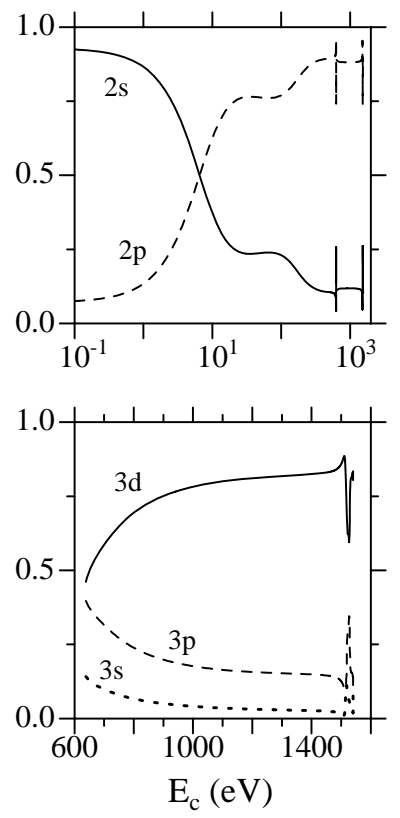

Figure 6. State distributions calculated with basis set B for $\left(H p^{-}\right)_{n=2}$ (upper panels) and $\left(H p^{-}\right)_{n=3}$ (lower panels) formation in collisions for (a) $p^{-}+\left(p \mu^{-}\right)_{1 s}$, (b) $p^{-}+\left(d \mu^{-}\right)_{1 s}$, and (c) $p^{-}+\left(t \mu^{-}\right)_{1 s}$. 
Table 1. Dipole moments $(\alpha<-1 / 4)$ of dipole states associated with $\mu^{-}+$ $\left(H p^{-}\right)_{n=2-4}$ fragmentations for partial-wave $J$, with $H=p, d, t$.

\begin{tabular}{cccc}
$J$ & $\mu^{-}+\left(p p^{-}\right)_{n}$ & $\mu^{-}+\left(d p^{-}\right)_{n}$ & $\mu^{-}+\left(t p^{-}\right)_{n}$ \\
\hline \multicolumn{3}{c}{$n=2$ manifold } \\
\cline { 2 - 4 } $\mathrm{S}$ & -0.624 & -0.398 & -0.330 \\
$\mathrm{P}$ & -0.261 & & \\
& \multicolumn{3}{c}{$n=3$ manifold } \\
\cline { 2 - 4 } $\mathrm{S}$ & -2.503 & -1.714 & -1.462 \\
$\mathrm{P}$ & -2.074 & -1.396 & -1.184 \\
$\mathrm{D}$ & -1.092 & -0.665 & -0.543 \\
& & $n=4$ manifold \\
$\mathrm{nyyy} \mathrm{S}$ & -5.673 & -4.021 & -3.486 \\
$\mathrm{P}$ & -5.225 & -3.678 & -3.180 \\
& -0.683 & & -2.522 \\
$\mathrm{D}$ & -4.269 & -2.941 & -1.360 \\
$\mathrm{~F}$ & -2.634 & -1.651 &
\end{tabular}

Table 2. Resonances in the $\left(p^{-}, H, \mu^{-}\right)$system, with $H=(p, d, t)$. Each resonance is expressed as $\left(E_{r}, \Gamma / 2\right)$ in $\mathrm{eV}$, where $E_{r}$ is the resonance energy measured from the associated threshold and $\Gamma$ is the width, with $x[y]=x \times 10^{y}$.

\begin{tabular}{|c|c|c|c|}
\hline$J$ & $\left(p^{-}, p, \mu^{-}\right)$ & $\left(p^{-}, d, \mu^{-}\right)$ & $\left(p^{-}, t, \mu^{-}\right)$ \\
\hline & \multicolumn{3}{|c|}{ Below the $\mu^{-}+\left(\mathrm{Hp}^{-}\right)_{n=2}$ threshold } \\
\hline \multirow{3}{*}{$\begin{array}{l}\mathrm{S} \\
\mathrm{P}\end{array}$} & $(-46.78,0.47)$ & $(-0.83,0.02)$ & $(-6.8[-3], 4[-4])$ \\
\hline & $(-0.10,0.04)$ & & \\
\hline & \multicolumn{3}{|c|}{ Below the $p^{-}+\left(H \mu^{-}\right)_{n=1}$ threshold } \\
\hline \multirow[t]{2}{*}{$\mathrm{S}$} & & & $(-15.3,6.1)$ \\
\hline & \multicolumn{3}{|c|}{ Below the $\mu^{-}+\left(H p^{-}\right)_{n=3}$ threshold } \\
\hline $\mathrm{S}$ & $(-12.47,1.6)$ & $(-3.10,0.87)$ & $(-1.5,0.5)$ \\
\hline $\mathrm{P}$ & $(-7.39,1.3)$ & $(-1.35,0.50)$ & $(-0.55,0.25)$ \\
\hline $\mathrm{D}$ & $(-0.65,0.15)$ & $(-0.015,9[-3])$ & $(-1.1[-3], 1.5[-3])$ \\
\hline & \multicolumn{3}{|c|}{ Below the $\mu^{-}+\left(H p^{-}\right)_{n=4}$ threshold } \\
\hline $\mathrm{S}$ & $(-82.80,2.6)$ & $(-37.91,3.8)$ & $(-25.49,3.8)$ \\
\hline $\mathrm{P}$ & $(-74.39,2.8)$ & $(-32.40,3.4)$ & $(-21.10,3.4)$ \\
\hline $\mathrm{D}$ & $(-56.69,2.0)$ & $(-20.15,2.3)$ & $(-11.92,2.1)$ \\
\hline $\mathrm{F}$ & $(-25.50,2.0)$ & $(-3.79,0.07)$ & $(-1.29,0.03)$ \\
\hline
\end{tabular}

